\title{
On rationality of verbal subsets in a group
}

\author{
A. Myasnikov, V. Roman'kov
}

March 22, 2011

\begin{abstract}
Let $F$ be a free non-abelian group. We show that for any group word $w$ the set $w[F]$ of all values of $w$ in $F$ is rational in $F$ if and only if $w[F]=1$ or $w[F]=F$. We generalize this to a wide class of free products of groups.
\end{abstract}

\section{Contents}

1 Introduction 1

2 Preliminaries 3

3 Free products and Rhemtulla's criterion

4 Positive elements 5

5 Free groups and free products

\section{Introduction}

In this paper we study the structure and complexity of the verbal sets in free groups and free products of groups. The main result of the paper shows that proper verbal subsets of free non-abelian groups are not rational. We generalize this to a wide class of free products of groups and other groups which have such free products as their quotients. In particular, the result holds for arbitrary finitely generated non-abelian residually free groups, pure braid groups, or nonabelian right angled Artin groups.

Following Gilman 8 we define for a given group $G$ the set $\operatorname{Rat}(G)$ of all rational subsets of $G$ as the closure of the set of all finite subsets of $G$ under the rational operations: union, product, and generation of a submonoid (Kleene's star operation). Sometimes, according to the standard practice, we refer to the rational subsets of a finitely generated free monoid as regular subsets. It is known (see [8]) that a subset $L$ of a group $G$ is rational in $G$ if and only if $L$ is accepted by a finite automaton over $G$ (see definitions in Section 2). 
Let $F(X)$ be a free group with a basis $X$ and $W \subseteq F(X)$ a subset of $F(X)$. An element $g$ in a group $G$ is called a $W$-element if $g$ is the image in $G$ of some word $w \in W$ under some homomorphism $F(X) \rightarrow G$. By $W[G]$ we denote the set of all $W$-elements in $G$. The set $W[G]$ generates the verbal subgroup $W(G)$. The verbal subgroups of groups were intensely studied in group theory especially with respect to relatively free groups and varieties of groups. We refer to the books [15, 21] for the general facts in this area.

$W$-width (or $W$-length) is one of the key notions concerning verbal subgroups $W(G)$ of a group $G$. The $W$-length $l_{W}(g)$ of an element $g \in W(G)$ is the the minimal natural number $n$ such that $g$ is a product of $n W$-elements in $G$ or their inverses. The $W$-width of the verbal subgroup $W(G)$ is $\sup \left\{l_{W}(g) \mid g \in W(g)\right\}$. It is usually assumed that the set $W$ is finite. In this case the set $W[G]$ is just a verbal set $w(G)$ for a suitable single word $w$. Furthermore, for a finite $W$ if the verbal subgroup $W(G)$ is of finite $W$-width then it is equal to $w[G]$ for some single word $w$ (not necessary from $W$ ). In particular, the length of $W(G)$ depends on the set $W$. From now on we will always assume that $W$ is just a singleton $W=\{w\}$ and refer to the related verbal set and the $W$ length as $w[G]$ and $w$-length. Usually, it is very hard to compute the $w$-length of a verbal subgroup $w(G)$ or the $w$-length of an element $g \in w(G)$. The first question of this type goes back to the Ore's paper [16] where he asked whether the commutator length (i.e., the $[x, y]$-length) of every element in a non-abelian finite simple group is equal to 1 (Ore Conjecture). Only recently the conjecture was established by Liebeck, O'Brian, Shalev and Tiep 12. For recent spectacular results on the $w$-length in finite simple groups we refer to the papers [11, 22] and a book [21.

Sometimes, for example in the presence of negative curvature, it is convenient to replace the unruly standard $W$-length with a more smooth stable $W$-length, which is defined for an element $g \in G$ as the $\operatorname{limit}_{n \rightarrow \infty} \frac{l_{W}(g)}{n}$ (which always exists). In some sense the stable commutator length relates to an $L^{1}$ filling norm with $\mathbb{Q}$ coefficients, introduced by Gromov in [9, see also Gersten's paper [7]. In 10] Gromov studied stable commutator length and its relation with bounded cohomology. We refer to a book [5] by Calegary on stable length of elements in groups.

On the other hand, Calegari showed in [6] that if a group $G$ satisfies a nontrivial law then the stable commutator length is equal to 0 for every element from $[G, G]$. In particular, in solvable groups the stable commutator length is not very helpful, instead, the standard $W$-length was studied intensely. We refer to papers [23, 19], 20, and a book [21] on the standard width of verbal subgroups in groups.

Properties of verbal sets $W[G]$ themselves play an important part in group theory. For example, the Membership Problem (MP) to the set $W[G]$ in $G$ is equivalent of solving in $G$ the homogeneous equations of the type $w(X)=g$, where $w \in W$ and $g \in G$. The Endomorphism Problem in $G$, which asks to decide for given elements $g, h \in G$ if there is an endomorphism $\phi \in \operatorname{End}(G)$ such that $\phi(g)=h$, is just a particular case of such a problem. Since the Diophantine 
Problem (of solving arbitrary equations) is decidable in a free group $F$ [14, MP to the verbal sets in $F$ is also decidable. However, Razborov showed in [17 that the solution sets of quadratic homogeneous equations of the type $\prod_{i=1}^{n}\left[x_{i}, y_{i}\right]=g$ may have a very complex structure.

In this paper we focus on the complexity of verbal sets in free groups from the formal language theory view point. The goal is to determine the proper place of various verbal sets of a free group $F$ (and some other "free-like" groups) in the hierarchy of formal languages. Since proper verbal subsets of $F$ are not rational in $F$, they are not regular in $F$, so they sit in some higher levels of the hierarchy of formal languages. What these levels are precisely is an interesting open problem.

The paper is organized as follows. Section 2 contains necessary definitions and facts about rational subsets in groups. In Section 3 we discuss Rhemtulla's gap theorem, which is the main technical tool of our approach to verbal sets in free groups and free products of groups. In Section 4 we establish some key lemmas on general properties of rational verbal subsets, while in Section 5 we prove the main results of the paper.

\section{Preliminaries}

Let $F=F(X)$ be a free group with basis $X=\left\{x_{1}, \ldots, x_{n}, \ldots\right\}$, viewed as the set of reduced words in $X \cup X^{-1}$ with the standard multiplication. Fix $w=w\left(x_{1}, x_{2}, \ldots, x_{n}\right) \in F(X)$. An element $g$ of a group $G$ is called a $w$-element if $g=w\left(g_{1}, g_{2}, \ldots, g_{n}\right)$ for some $g_{1}, \ldots, g_{n} \in G$. We denote the set of all $w$ elements of $G$ by $w[G]$. A subset $M \subseteq G$ is a verbal subset of $G$ if $M=w[G]$ for some word $w \in F(X)$. The subgroup $w(G)$ generated by $w[G]$ is the $w$-verbal subgroup of $G$, and a subgroup of $G$ is called verbal if it is equal to $w(G)$ for some $w$.

A word $w$ is said to be proper if there exist groups $G$ and $H$ such that $w[G] \neq 1$ and $w[H] \neq H$, in fact, in this case $1 \neq w[G \times H] \neq G \times H$.

Any element $w=w\left(x_{1}, \ldots, x_{n}\right) \in F(X)$ can be written as a product

$$
w=x_{1}^{t_{1}} x_{2}^{t_{2}} \ldots x_{n}^{t_{n}} w^{\prime}
$$

where $w^{\prime}=w^{\prime}\left(x_{1}, \ldots, x_{n}\right) \in[F, F]$. Since the exponents $t_{1}, t_{2}, \ldots, t_{n}$ depend only on the element $w$ the number $e(w)=g c d\left(t_{1}, t_{2}, \ldots, t_{n}\right)$ is well-defined (here we put $e(w)=0$ if $\left.t_{1}=\ldots=t_{n}=0\right)$. If $e(w)=0$ then we refer to $w$ as a commutator word. A non-trivial commutator word is obviously proper. If $e(w)>0$ then there exist integers $r_{1}, r_{2}, \ldots, r_{n}$ such that $\sum_{i=1}^{n} r_{i} t_{i}=e$, so for an arbitrary group $G$ and an element $g \in G$ one has $w\left(g^{r_{1}}, g^{r_{2}}, \ldots, g^{r_{n}}\right)=g^{e}$. In particular, $e(w)=1$ implies that $w[G]=G$ for every group $G$, so $w$ is not proper. If $e(w)>1$ then $w$ is proper, which can be seen in an infinite cyclic group. In other words, a non-trivial word $w$ is proper if and only if $e(w) \neq 1$.

Let $M$ be a monoid. For $L \subseteq M$ by $L^{*}$ we denote the submonoid of $M$ generated by $L$. The set $\operatorname{Rat}(M) \subseteq 2^{M}$, of all rational subsets of $M$, is defined as the smallest (with respect to inclusion) subset which contains all finite subsets 
of $M$ and closed under the following operations (here $L_{1}, L_{2}, L$ are subsets of $M)$ :

- Union: $\left(L_{1}, L_{2}\right) \rightarrow L_{1} \cup L_{2}$.

- Product: $\left(L_{1}, L_{2}\right) \rightarrow L_{1} L_{2}=\left\{a b \mid a \in L_{1}, b \in L_{2}\right\}$.

- Submonoid generation: $L \rightarrow L^{*}$.

It follows from the definition above that every rational set $L \in \operatorname{Rat}(M)$ in a monoid $M$ can be presented in a form

$$
L=\cup_{i=1}^{k} a_{i 1} E_{i 1}^{*} \ldots a_{i t_{i}} E_{i t_{i}}^{*} a_{i, t_{i}+1}
$$

where all coefficients $a_{i j}$ are in $M$ and each $E_{i j}$ is a rational subset of $M$.

We define a complexity function $c: \operatorname{Rat}(M) \rightarrow \mathbb{N}$ as follows. Put $c(L)=0$ if and only if $L$ is finite. Suppose now that rational sets $L \in \operatorname{Rat}(M)$ with $c(L) \leq n-1$ are defined. Then for a set $L \in \operatorname{Rat}(M)$ we put $c(L)=n$ if and only if $c(L) \not \leq n-1$, but either $L=L_{1} \cup L_{2}$, or $L=L_{1} L_{2}$, or $L=L_{1}^{\star}$, for some $L_{i} \in \operatorname{Rat}(M)$ with $c\left(L_{i}\right) \leq n-1, i=1,2$.

It is easy to see that if $G$ is a group then for any element $g \in G$ and a set $L \in \operatorname{Rat}(G)$ one has

$$
c\left(g^{-1} L g\right)=c(L) .
$$

A finite $M$-automaton is a tuple $A=\left(Q, \delta, q_{0}, F\right)$ where

- $Q$ is a finite set of states,

- $q_{0} \in Q$ is the initial state,

- $F \subseteq Q$ is the set of terminating states,

- $\delta \subseteq Q \times M \times Q$ is a finite relation, termed the transition relation.

One can view an automaton $A=\left(Q, \delta, q_{0}, F\right)$ as a directed $M$-labelled graph (possibly with multiple edges), with the set of vertices $Q$ and where two vertices $u, v$ are connected by an edge $u \rightarrow v$ labelled by $m \in M$ if and only if $(u, m, v) \in$ $\delta$. As usual, a path $p$ in $A$ from a vertex $u$ to a vertex $v$ is a sequence of edges

$$
\left(u_{0}, m_{1}, u_{1}\right),\left(u_{1}, m_{2}, u_{2}\right), \ldots,\left(u_{k-1}, m_{k}, u_{k}\right)
$$

such that $u=u_{0}$ and $v=u_{k}$. The label $\lambda(p)$ of $p$ is the product $m_{1} \ldots m_{k} \in M$. A successful path is a path from $q_{0}$ to a vertex in $F$. as

The subset $L(A) \subseteq M$ (the set of all elements in $M$ accepted by $A$ ) is defined

$$
L(A)=\{\lambda(p) \mid p \text { is a successful path in } A\} .
$$

It was shown in [8] that for any monoid $M$ and any subset $L \subseteq M$ the following equivalence holds:

$$
L \in \operatorname{Rat}(M) \Longleftrightarrow L=L(A) \text { for some automaton } A \text { over } M \text {. }
$$


Gilman showed in [8] that any rational subset $L$ of a group $G$ generates in $G$ a finitely generated (and so rational) subgroup. Hence if a verbal set $w[G]$ is rational then the verbal subgroup $w(G)$ is finitely generated. In the case when $G$ is a free non-abelian group, or more generally, a non-trivial free product $G=A * B$, every normal subgroup of infinite index is not finitely generated (see [13] and [3]), and so it is not rational. Hence the case when the verbal subgroup $w(G)$ has finite index in $G$ becomes the most interesting in our study.

\section{Free products and Rhemtulla's criterion}

Let $G=A * B$ be a free product of non-trivial groups $A$ and $B$. Each element $u \neq 1$ of $G$ can be uniquely written in its reduced form $u=u_{1} u_{2} \ldots u_{m}$, where $u_{i} \in A \cup B \backslash\{1\}(i=1,2, \ldots, m)$; and for every $i$ the elements $u_{i}, u_{i+1}$ are from different groups $A$ and $B$. The number $m$ is the syllable length of $u$, denoted by $|u|$. Put $\operatorname{supp}(u)=\left\{u_{1}, \ldots, u_{m}\right\}$. Furthermore, the reduced form of a non-trivial element $u \in G$ can be uniquely written as

$$
u=r_{t}^{-1} \ldots r_{1}^{-1} v_{1} \ldots v_{k} r_{1} \ldots r_{t},
$$

where either $k=1$, or $k>1$ and $v_{1} v_{k} \neq 1$. We refer to $v_{1} \ldots v_{k}$ as the core of $u$ and denote it by $\bar{u}$.

As usual one can define a cyclically reduced form $u^{0}$ of an element $u \in$ $G$. Namely, let the core $\bar{u}$ of $u$ is given in the reduced form $\bar{u}=u_{1} u_{2} \ldots u_{m}$. Then if $u_{1}, u_{m}$ are from the different factors then $u^{0}=u$. Otherwise, $u^{0}=$ $\left(u_{2} \ldots u_{m-1},\left(u_{m} u_{1}\right)\right)$.

In [18] A.H. Rhemtulla introduced a useful technique of gap functions for $G=A * B$. To explain, suppose one of the groups, say $B$, has an element $b \in B$ such that $b \neq b^{-1}$. Let $u=u_{1} u_{2} \ldots u_{m}$ be a non-trivial element in $G$ given in its reduced form. A subsequence $u_{i}, u_{i+1}, \ldots, u_{i+2 k}$ of the reduced form of $u$ is called a $b-g a p$ in $u$ of length $2 k-1$ if $u_{i}=u_{i+2 k}=b$ and $u_{j} \neq b$ for any $i<j<i+2 k$.

For $k=1,2, \ldots$ denote by $\delta_{b, k}(u)$ the number of $b$-gaps of length $2 k-1$ in $u$. For a positive integer $e$ put $\gamma_{b, e}(u)$ to be the number of values $k$ such that $\delta_{b, k}(u) \neq \delta_{b^{-1}, k}(u)(\bmod e)$.

Rhemtulla's criterion [18]. Let $G=A * B$ and $b \in B$ as above. Then for any word $w\left(x_{1}, \ldots, x_{n}\right)$ with $e=e(w)>1$ the function $\gamma_{b, e}$ is bounded on the set $w[G]$.

\section{Positive elements}

A sign function on a group $G$ is a function $\rho: G \rightarrow\{-1,1\}$ such that:

- $\rho(1)=1$;

- for any $f, g \in G$ if $\rho(f)=1, \rho(g)=1$ then $\rho(f g)=1$. 
The set of positive elements $\operatorname{Pos}(G)=\{g \in G \mid \rho(g)=1\}$ forms a submonoid in $G$. Conversely, a group $G$ with a distinguished submonoid $M \subseteq G$ admits a sign function $\rho_{M}$ such that $\rho_{M}(g)=1$ if and only if $g \in M$. We refer to groups with sign functions as s-groups. Elements from $M$ are called positive, all others - negative. The following examples are important in our context.

Example 4.1. Let $C=\langle a\rangle$ be an infinite cyclic group generated by $a$. Then $\rho\left(a^{n}\right)=1$, if $n \geq 0$, and $\rho\left(a^{n}\right)=-1$ if $n<0$ is a sign function.

Generalizing the example above, we get the following

Example 4.2. Let $G$ be a group with a generating set $X$. Then the submonoid $\operatorname{mon}(X)=X^{*}$ generated by $X$ gives a sign function $\rho_{X}$ on $G$.

Example 4.3. Let $G=A * B$ be a free product of two non-trivial $s$-groups $A, B$. The function $\rho: G \rightarrow\{-1,1\}$ such that $\rho(g)=1$ if and only if all factors in the reduced form of $g \in A * B$ are positive, is a sign function on $G$, termed the standard free product sign function.

When applied to a free group $F$ with basis $X$ the examples above give the standard notion of a positive word in $F$.

Notice, that there might be "zero divisors" in $G$ relative to $\operatorname{Pos}(G)$, i.e., some elements $x, y \in G$, not both positive, such that $x y \in \operatorname{Pos}(G)$. For example, if $u \in G$ is negative then $u u^{-1}$ is positive. To separate the natural cases like $u u^{-1}$ above (which is easy to deal with) from the harder ones we introduce the following notion. We say that a sign function $\rho$ on $G$ is reduced if it has the following property:

- for any two subsets of elements $S, T$ of $G$ if $S T \subseteq \operatorname{Pos}(G)$ then here exists an element $u \in G$ such that $S u^{-1} \subseteq \operatorname{Pos}(G)$ and $u T \subseteq \operatorname{Pos}(G)$.

In particular, if $u T \subseteq \operatorname{Pos}(G)$ then there is an element $u_{0}$ such that $u u_{0}^{-1} \in$ $\operatorname{Pos}(G)$ and $u_{0} T \subseteq \operatorname{Pos}(G)$.

Notice also, that if $\rho$ is reduced then for a fixed set $S$ there is a single $u$ that works for all subsets $T$ as above; a similar claim holds for a fixed set $T$.

We say that a sign function $\rho$ on $G$ is strongly reduced if it is reduced and has the following property:

- any product of two negative elements is negative.

Remark 4.4. The sign function on the infinite cyclic group from Example 4.1 is strongly reduced.

Lemma 4.5. The standard sign function (see Example 4.3) on a free product $G=A * B$ of two non-trivial s-groups with reduced sign functions is reduced. In particular, the standard sign function on a free group is reduced.

Proof. Let $S, T$ be non-empty subsets of $G$ such that $S T \subseteq P o s(G)$. For an element $u \in S \backslash \operatorname{Pos}(G)$ written in its reduced form $u=u_{1} u_{2} \ldots u_{i} \ldots u_{k}$ denote 
by $i=i(u)$ such an index that $u_{i}$ is negative, but all the factors $u_{1}, \ldots, u_{i-1}$ are positive. Similarly, for an element $v=v_{1} v_{2} \ldots v_{j} \ldots v_{l} \in T \backslash \operatorname{Pos}(G)$ written in its reduced form let $j=j(v)$ be the index such that $v_{j}$ is negative but all the factors $v_{j+1}, \ldots, v_{l}$ are positive. Notice, that the sets $I=I(S)=\{|u|-i(u)+1 \mid u \in$ $S \backslash \operatorname{Pos}(G)\}$ and $J=J(T)=\{j(v) \mid v \in T \backslash \operatorname{Pos}(G)\}$ are bounded even if the sets $S, T$ are infinite. Indeed, if, say, $J$ is unbounded then for a given $u \in S$ there exists $v^{\prime} \in T$ with $|u|<j\left(v^{\prime}\right)$, in which case $u v^{\prime}$ is not positive, - contradicting the hypotheses of the lemma. Put $\max (\emptyset)=0$, and $i_{0}=\max (I), j_{0}=\max (J)$. The case $S, T \subseteq \operatorname{Pos}(G)$ when $I=J=\emptyset$ and $i_{0}=j_{0}=0$ is obvious. Assume that $i_{0} \leq j_{0}$ (the other case can be treated similarly). Let $\tilde{v} \in T \backslash \operatorname{Pos}(G)$ be an element with $j_{0}=j(v)$. Write $\tilde{v}$ in the reduced form $\tilde{v}=v_{1} \ldots v_{j_{0}-1} v_{j_{0}} \ldots v_{l}$, and assume that $v_{j_{0}}$ lies, say, in $B$. Denote $c=v_{1} \ldots v_{j_{0}-1}$. Then for every element $u \in S$ the factor $v_{j_{0}-1}$ cancels out in the reduced form of $u v$ (otherwise a negative factor $v_{j_{0}}$ occurs in the reduced form of $u v$ ). Hence the reduced form of each $u \in S$ is of the type $u=u_{1} \ldots u_{r(u)} c^{-1}$ for a suitable index $r(u)$ and $u_{r(u)} \in B$. Consider now two cases.

Case 1. $i_{0}=j_{0}$. Let $\tilde{u} \in S \backslash \operatorname{Pos}(G)$ be an element with $i_{0}=i(u)$. In this case $\tilde{u}=\tilde{u}_{1} \ldots \tilde{u}_{r(\tilde{u})} c^{-1}$, where $\tilde{u}_{r(\tilde{u})} \in B$ is negative. The argument above shows that every element $v \in T$ can be written in the reduced form $v=c v_{j_{0}} \ldots v_{m}$, where $v_{j_{0}} \in B$. It follows that for any $u \in S, v \in T$ one has $u v=u_{1} \ldots\left(u_{r(u)} v_{j_{0}}\right) \ldots v_{m}$, where all the factors, including $\left(u_{r(u)} v_{j_{0}}\right)$, are positive. Since the sign function in $B$ is reduced there is an element $b \in B$ such that the elements $u_{r(u)} b^{-1}, b v_{j_{0}}$ are positive in $B$ for any $u \in S, v \in T$. Hence, $S c b^{-1} \subseteq \operatorname{Pos}(G)$ and $b c^{-1} T \subseteq P o s(G)$, as required.

Case 2. $i_{0}<j_{0}$. In this case the reduced form of each $u \in S$ is of the type $u=u_{1} \ldots u_{r(u)} c^{-1}, u_{r(u)} \in B$, and furthermore, $u_{r(u)} \in \operatorname{Pos}(B)$. Observe that for every $v \in T$ since $u v \in \operatorname{Pos}(G)$ then in the product $c^{-1} v$ either $c^{-1}$ cancels out completely or $c^{-1} v \in \operatorname{Pos}(G)$. In the former case $v=c v^{\prime}$ and the reduced form of $v^{\prime}$ is of the type $v_{1}^{\prime} \ldots v_{m}^{\prime}$; in this event put $y(v)=v_{1}^{\prime}$. In the latter case, put $y(v)=1$. By construction the sets $S_{B}=\left\{u_{r(u)} \mid u \in S\right\} \subseteq B$ and $T=\{y(v) \mid v \in T\} \subseteq B$ are such that $S_{B} T_{B} \subseteq \operatorname{Pos}(B)$. Hence there is $b \in B$ with $S_{B} b^{-1} \subseteq \operatorname{Pos}(\bar{B}), b T_{B} \subseteq \operatorname{Pos}(B)$. It follows that $S c b^{-1} \in \operatorname{Post}(G)$ and $b c^{-1} T \subseteq \operatorname{Pos}(G)$ as claimed.

Lemma 4.6. Let $A, B$ be non-trivial groups with strongly reduced sign functions and $G=A * B$ equipped with the standard free product sign function. If $L \in \operatorname{Rat}(G)$ and for some elements $u, v \in G u L v \subseteq \operatorname{Pos}(G)$ then $u L v \in \operatorname{Rat}(\operatorname{Pos}(G))$.

Proof. We use induction on $c(L)$. If $c(L)=0$ then $L$ is finite and the claim is obvious. Now consider the following cases.

Case 1) If $L=L_{1} \cup L_{2}$ and $c\left(L_{i}\right)<c(L)$ for $i=1,2$ the result follows by induction.

Case 2) Suppose $L=L_{1} L_{2}$ and $c\left(L_{i}\right)<c(L)$ for $i=1,2$. Then $u L v$ is a product of two rational sets $u L_{1}$ and $L_{2} v$. Since the standard free product sign function on $A * B$ is reduced there is an element $w \in G$ for which 
$u L_{1} w^{-1}, w L_{2} v \subseteq \operatorname{Pos}(G)$. Then $u L_{1} w^{-1}, w L_{2} v \in \operatorname{Rat}(\operatorname{Pos}(G))$ by induction, hence $u L v=u L_{1} w^{-1} \cdot w L_{2} v \in \operatorname{Rat}(\operatorname{Pos}(G))$.

Case 3) Suppose $L=L_{1}^{*}$, where $c\left(L_{1}\right)<c(L)$. Then $u L v=u L_{1}^{*} v=$ $u v\left(v^{-1} L_{1} v\right)^{*}$, and $w=u v \in \operatorname{Pos}(G)$ since $1 \in L_{1}^{*}$. Denote $L_{2}=v^{-1} L_{1} v$, so $u L v=w L_{2}^{*}$ and $c\left(L_{2}\right)=c\left(v^{-1} L_{1} v\right)=c\left(L_{1}\right)<c(L)$.

If $L_{2} \subseteq \operatorname{Pos}(G)$ then the result follows by induction. Otherwise, there is an element $l \in L_{2} \backslash \operatorname{Pos}(G)$. If $l=l_{1} \ldots l_{i} l_{i+1} \ldots l_{t}$ is the reduced form of $l$ then there exists $i$ such that $l_{i}$ is negative and all the factors $l_{i+1}, \ldots, l_{t}$ are positive. Suppose that the reduce form of $l$ is written in the form

$$
l=r_{t}^{-1} \ldots r_{1}^{-1} u_{1} \ldots u_{k} r_{1} \ldots r_{t},
$$

where either $k=1$, or $k>1$ and $u_{1} u_{k} \neq 1$.

Claim 1. In the notation above the factor $l_{i}$ of $l$ is among the first $t+1$ factors $r_{t}^{-1}, \ldots, r_{1}^{-1}, u_{1}$, i.e., $i \leq t+1$.

Indeed, the reduced form of every element $d \in w L_{2}^{*}$ must have the product $l_{i-1}^{-1} \ldots l_{1}^{-1}$ at the end, otherwise the element $d l \in w L_{2}^{*}$ would not be positive.

If $i>|l| / 2$ then for every $d \in w L_{2}^{*}$ more then half of $l$ cancels out in $d l$, so $|d l|<|d|$. This implies that $w L_{2}^{*}$ does not have an element of minimal length, i.e., $w L_{2}^{*}=\emptyset$, which contradicts the fact that $w \in w L_{2}^{*}$.

Assume now that $i \leq|l| / 2$, but $i>t+1$. It follows that $k \geq 2$. In this case for any natural number $p$ one has $l^{p} \in L_{2}^{*}$ and the rightmost occurrence of the negative factor $l_{i}$ of $l$ does not cancel in $l^{p}$ (since it is in the core $\bar{l}$ of $l$ ). Therefore, for sufficiently large $p$ the rightmost occurrence of the factor $l_{i}$ does not cancel in $w l^{p}$, so the element $w l^{p}$ is negative, which contradicts the condition $w L_{2}^{*} \subseteq \operatorname{Pos}(G)$. Hence $i \leq t+1$ and the claimed follows.

Let's take $l \in L_{2} \backslash \operatorname{Pos}(G)$ such that $i=i(l)$ be the maximal possible such index among all elements $l$ in $L_{2} \backslash \operatorname{Pos}(G)$ (such $i$ exists since $w L_{2}^{*} \subseteq \operatorname{Pos}(G)$ ). We can also assume that $l_{i}=b \in B$. It follows that the reduced form of $w$ is equal to $w^{\prime} b(w) l_{i-1}^{-1} \ldots l_{1}^{-1}$, where $b(w) \in B$ and $b(w) b \in \operatorname{Pos}(B)$.

Claim 2. The reduced form of any element $d \in L_{2}$ can be written as $d=$ $d^{\prime} b(d) l_{i-1}^{-1} \ldots l_{1}^{-1}$, where $b(d) \in B$ and $b(d) b \in \operatorname{Pos}(B)$.

Indeed, suppose $m \in L_{2}$ does not have $l_{i-1}^{-1} \ldots l_{1}^{-1}$ at the end. Then $m=$ $m_{1} \ldots m_{s} l_{q}^{-1} \ldots l_{1}^{-1}$, where $0 \leq q<i-1$ (we assume $m=m_{1} \ldots m_{s}$ for $q=0$ ) and $m_{s} l_{q+1} \neq 1$ (notice that $m_{s}$ and $l_{q+1}$ are in the same factor). Since $w m l$ is positive the negative factor $l_{i}$ of $l$ cancels out in $w m l$, so $m_{1} \ldots m_{s-1}\left(m_{s} l_{q+1}\right)$ must cancel out in $w m$. The element $w$ ends on $l_{i-1}^{-1} \ldots l_{1}^{-1}$. If $s>q$ then $m=$ $l_{1} \ldots l_{q} m_{q+1} \ldots m_{s} l_{q}^{-1} \ldots l_{1}^{-1}$, and $m_{q+1}=l_{q+1}$. On the other hand $m_{s} l_{q+1} \neq 1$, which shows that $m_{q+1} \ldots m_{s}$ is the core of $m$. If $s \neq q+1$ then the length of the core of $m$ is greater than 1, so for sufficiently large integer $p$ the element $w m^{p} l \in w L_{2}^{*}$ is negative - contradiction. If $s=q+1$ then $m=l_{1} \ldots l_{q} l_{q+1} l_{q}^{-1} \ldots l_{1}^{-1}$ and $w m l=w^{\prime} l_{q+1}^{-1} l_{q+1} l_{q+1} l_{q+2} \ldots l_{t}$, whose reduced form is $w^{\prime} l_{q+1} l_{q+2} \ldots l_{t}$, so it contains $l_{i}$ - contradiction. The case $s \leq q$ can be done similarly. This proves the claim.

In the notation above, since the sign function on $B$ is strongly reduced there is a positive element $b_{0} \in B$ for which $b_{0} b, b(w) b_{0}^{-1}$ and $b(d) b_{0}^{-1}$ are positive 
for all $d \in L_{2}$. Denote $r=b_{0} l_{i-1}^{-1} \ldots l_{1}^{-1}$. Notice, that $r$ is positive. It follows from Claim 2 that every element $d \in L_{2}$ can be written in the reduced form as $d=d^{\prime}\left(b(d) b_{0}^{-1}\right) r$. Let

$$
L_{3}=\left\{d^{\prime}\left(b(d) b_{0}^{-1}\right) \mid d \in L_{2}\right\}
$$

Claim 3. The language $r L_{3}$ is positive.

Indeed, it follows from the argument above.

Notice that $r L_{3}=r L_{2} r^{-1}$ so $r L_{3}$ is rational and $c\left(r L_{3}\right)=c\left(L_{2}\right)<c(L)$. Hence by induction $r L_{3} \in \operatorname{Rat}(\operatorname{Pos}(G))$. Now

$$
w L_{2}^{*}=w^{\prime} r\left\{L_{2}\right\}^{*}=w^{\prime}\left\{r L_{3}\right\}^{*} r,
$$

where all factors in product on the right are positive. Hence $u L v=w L_{2}^{*} \in$ $\operatorname{Rat}(\operatorname{Pos}(G))$, which proves the lemma.

Lemma 4.7. Let $A, B$ be non-trivial groups with strongly reduced sign functions and $G=A * B$ equipped with the standard sign function. If $L \in \operatorname{Rat}(G)$ and $L \subseteq \operatorname{Pos}(G)$ then $L \in \operatorname{Rat}(\operatorname{Pos}(G))$.

Proof. We use induction on complexity $c(L)$ of $L$. If $c(L)=0$ then $L$ is finite and the claim is obvious. Consider the following cases.

Case 1) If $L=L_{1} \cup L_{2}$ or $L=L_{1}^{*}$ with $c\left(L_{i}\right)<c(L), i=1,2$, then $L_{1}, L_{2} \subseteq$ $\operatorname{Pos}(G)$ and the result follows by induction.

Case 2) Suppose $L=L_{1} L_{2}$. By Lemma 4.5 the standard free product sign function on $G$ is reduced. Therefore there is $u \in G$ such that $L_{1} u, u^{-1} L_{2} \subseteq \operatorname{Pos}(G)$. By Lemma 4.6 $L_{1} u, u^{-1} L_{2} \subseteq \operatorname{Rat}(\operatorname{Pos}(G))$. Hence $L=\left(L_{1} u\right)\left(u^{-1}\right) L_{2} \subseteq \operatorname{Rat}(\operatorname{Pos}(G))$, as claimed.

Lemma 4.8. Let $A, B$ be two non-trivial groups with strongly reduced sign functions and such that the sets $\operatorname{Rat}(A), \operatorname{Rat}(B)$ are closed under intersections and complements (form Boolean algebras) and $G=A * B$. If the submonoid Pos $(G)$ relative to the standard sign function on $G$ is rational then for any $\bar{L} \in \operatorname{Rat}(G)$ the intersection $L=\bar{L} \cap \operatorname{Pos}(G)$ is rational in the monoid $\operatorname{Pos}(G)$.

Proof. Observe, that under the premises of the theorem $\operatorname{Rat}(G)$ is a Boolean algebra by G.A. Bazhenova's result [1], which states that class of groups with Boolean algebras of rational subsets is closed under free products. Hence $L$ is rational in $G$. Then $L \in \operatorname{Rat}(\operatorname{Pos}(G))$ by Lemma 4.7.

Corollary 4.9. Let $F_{2}=F\left(X_{2}\right)$ be a free non-abelian group of rank 2 with basis $X_{2}=\left\{x_{1}, x_{2}\right\}$. Denote by $X_{2}^{*}$ the free submonoid of $F_{2}$ generated by $X_{2}$. If $\bar{L} \in \operatorname{Rat}\left(F_{2}\right)$ then $L=\bar{L} \cap X_{2}^{*} \in \operatorname{Rat}\left(X_{2}^{*}\right)$.

Proof. Follows from Lemma 4.8 . 


\section{$5 \quad$ Free groups and free products}

Let $F=F(X)$ be a free non-abelian group with basis $X=\left\{x_{1}, x_{2}, \ldots\right\}$. Put $X_{2}=\left\{x_{1}, x_{2}\right\}$ and consider the free group $F_{2}=F\left(X_{2}\right)$ with basis $X_{2}$ as the distinguished subgroup of $F(X)$ generated by $X_{2}$. By $X_{2}^{*}$ we denote the free submonoid of $F_{2}$ generated by $X_{2}$.

Lemma 5.1. Let $G=A * B$ be a free product of two non-trivial groups $A$ and $B$. If $w$ is a proper word such that the verbal subgroup $w(G)$ has infinite index in $G$ then the set $w[G]$ is not rational in $G$.

Proof. Observe that for such $w$ the verbal subgroup $w(G)$ is a non-trivial normal subgroup of infinite index in $G$. By B. Baumslag's result [3] the subgroup $w(G)$ is not finitely generated. Since a subgroup generated in a group by a rational subset has to be finitely generated (see [8], Theorem 4.2), the generating set $w[G]$ of $w(G)$ is not rational in $G$.

Corollary 5.2. Let $G=A * B$ be a free product of non-trivial groups $A$ and $B$ with infinite abelianization $G_{a b}=G /[G, G]$. Then for any non-trivial commutator word $w$ the set $w[G]$ is not rational in $G$.

Corollary 5.3. Let $F$ be a free non-abelian group. Then for any non-trivial commutator word $w$ the set $w[F]$ is not rational in $F$.

For the rest of the paper we fix a proper non-commutator word $w$. Observe, that $e=e(w) \geq 2$. To apply Rhemtulla's criterion we view the free group $F_{2}$ as a free product $F_{2}=\left\langle x_{1}\right\rangle *\left\langle x_{2}\right\rangle=A * B$, equipped with the standard sign function given by the submonoid $X_{2}^{*}$. This sign function is strongly reduced.

Lemma 5.4. Let $p, q \in X_{2}^{*}$ and $E \subseteq X_{2}^{*}$ be such that $p E^{*} q \subseteq w\left[F_{2}\right]$. Then one of the following hold:

1) $|u| \geq 2$ for every $u \in E^{*}$. In this case $\operatorname{supp}\left(p E^{*} q\right)$ is finite.

2) $|u|=1$ for every $u \in E^{*}$. In this case either $E^{*} \subseteq x_{1}^{*}$ or $E^{*} \subseteq x_{2}^{*}$.

Proof. Let $u, v \in E^{*}$. Since $E \subseteq X_{2}^{*}$ the elements $u, v$ are positive, so they are equal to their cores $u=\bar{u}, v=\bar{v}$. Assume that $\bar{u}, \bar{v}$ are given in the reduced forms

$$
\bar{u}=u_{1} u_{2} \ldots u_{k}, \quad \bar{v}=v_{1} v_{2} \ldots v_{l} .
$$

We prove first that if $k, l \geq 2$ then $\operatorname{supp}\left(u^{0}\right)=\operatorname{supp}\left(v^{0}\right)$. Notice, that either $\operatorname{supp}\left(u^{0}\right)=\left\{u_{1}, u_{2}, \ldots, u_{k}\right\}$ for even $k$, or $\operatorname{supp}\left(u^{0}\right)=\left\{u_{2}, u_{3}, \ldots,\left(u_{k} u_{1}\right)\right\}$ for odd $k$.

Since

$$
\operatorname{puu}\{v\}^{*} u u q \subseteq X_{2}^{*} \cap w\left[F_{2}\right]
$$

the Rhemtulla's criterion shows that $\operatorname{supp}\left(u^{0}\right) \subseteq \operatorname{supp}\left(v^{0}\right)$. Indeed, $|\bar{v}| \geq 2$ implies that the length of $v^{n}$ strictly grows with $n$, so if $b$ is a factor in $\operatorname{supp}\left(u^{0}\right)$ but not in $\operatorname{supp}\left(v^{0}\right)$ then for infinitely many $k$ there is a number $n=n(k)$ such 
that the word puuv ${ }^{n} u u q$ contains precisely one $b$-gap of length $k$. So the gap function $\delta_{b, k}(u)$ is equal to 1 for infinitely many $k$ on $p u u\{v\}^{*} u u q$. Notice that $\delta_{b^{-1}, k}\left(\right.$ puuv $\left.^{n} u u q\right)=0$ since the words are positive. Hence the function $\gamma_{b, e}$ is unbounded on $w\left[F_{2}\right]$ - contradicting the Remtulla's criterion. Similarly, we show that $\operatorname{supp}\left(v^{0}\right) \subseteq \operatorname{supp}\left(u^{0}\right)$. Hence $\operatorname{supp}\left(u^{0}\right)=\operatorname{supp}\left(v^{0}\right)$ as claimed.

Observe now that if there are elements $u, v \in E$ such that $|u| \geq 2$ and $|v|=1$ then for sufficiently large $n$ one has $|u|,\left|u v^{n}\right| \geq 2$ and $\operatorname{supp}\left(u^{0}\right) \neq \operatorname{supp}\left(\left(u v^{n}\right)^{0}\right)$ - contradicting the statement above.

The argument above shows that either all elements in $E^{*}$ are of syllable length greater then 1 , or all of them have length 1 . This proves 2) and the first part of 1). To finish the proof observe that for any $u=u_{1} \ldots u_{k} \in E^{*}$ one has $u_{2}, \ldots, u_{k-1}, u_{k} u_{1} \in \operatorname{supp}\left(v^{0}\right)$. Since $u_{1}, u_{k}, u_{1} u_{k}$ are positive there are only finitely many choices for $u_{1}$ and $u_{k}$ as divisors of $u_{k} u_{1}$. This proves that there is a finite set $K \subseteq X_{2}^{*}$ such that for any $u \in E^{*} \operatorname{supp}(u) \subseteq K$. It follows that $\operatorname{supp}\left(p E^{*} q\right)$ is finite as claimed.

Corollary 5.5. Let $L \in \operatorname{Rat}\left(X_{2}^{*}\right) \cap w\left[F_{2}\right]$. Then there is a finite set $K_{L} \subseteq x_{1}^{*} \cup x_{2}^{*}$ and a natural number $n=n(L)$ such that every element $u \in L$ can be presented as a product of the following type:

$$
u=s_{1} t_{1} s_{2} \ldots s_{n} t_{n}
$$

where $\operatorname{supp}\left(s_{i}\right) \subseteq K_{L}$ and $t_{i} \in x_{1}^{*} \cup x_{2}^{*}$.

Proof. Since $L \in \operatorname{Rat}\left(X_{2}^{*}\right)$ it can be presented in the form

$$
L=a_{i 1} E_{i 1}^{*} \ldots a_{i j} E_{i j}^{*} a_{i j+1} \ldots E_{i t_{i}}^{*} a_{i t_{i}+1}
$$

For $E_{i j}$ as above, put $E=E_{i j}$ and denote $p=a_{i 1} \ldots a_{i j}, q=a_{i j+1} \ldots a_{i t_{i}+1} \in$ $X_{2}^{*}$. Since each $E_{i l}$ contain 1 we have $p E^{*} q \subseteq \operatorname{Rat}\left(X_{2}^{*}\right) \cap w\left[F_{2}\right]$. Now the result follows from Lemma 5.4

Theorem 5.6. Let $F$ be a free non-abelian group and $w$ be a proper word. Then the set $w[F]$ is not rational in $F$.

Proof. Let $w$ be a proper word such that $w[F]$ is rational in $F$. Notice that $e=e(w) \geq 2$ since $w$ is proper. By Corollary 5.3 the word $w$ is not a commutator word. Observe that $\bar{L}=w[F] \cap F_{2}=w\left[F_{2}\right]$ is rational in $F_{2}$ as a homomorphic image of a rational set under standard homomorphism $F \rightarrow F_{2}$. By Corollary 4.9 the set $L=\bar{L} \cap X_{2}^{*}$ is rational in $X_{2}^{*}$. By Corollary 5.5 there is a finite set $K_{L} \subseteq x_{1}^{*} \cup x_{2}^{*}$ and a natural number $n=n(L)$ such that every element $u \in L$ can be presented as a product of the following type:

$$
u=s_{1} t_{1} s_{2} \ldots s_{n} t_{n},
$$

where $\operatorname{supp}\left(s_{i}\right) \subseteq K_{L}$ and $t_{i} \in x_{1}^{*} \cup x_{2}^{*}$. Chose $t \in \mathbb{N}$ large enough so $x_{1}^{t} \notin K_{L}$. Chose $l \in \mathbb{N}$ such that $l>n(L)$. Then the word $u=\left(x_{1}^{t} x_{2}\right)^{l e}$ belongs to $w\left[F_{2}\right]$, hence it belongs to $L$. However, $u$ cannot be presented in the form (5) contradiction, which proves the theorem. 
Theorem 5.6 can be generalized into free products as follows.

Theorem 5.7. Let $A$ and $B$ be groups containing elements of infinite order $x_{1} \in A, x_{2} \in B$, and $G=A * B$. If the rational sets $\operatorname{Rat}(A)$ and $\operatorname{Rat}(B)$ are Boolean algebras then for every proper word $w$ with $e(w) \geq 2$ the set $w[G]$ is not rational in $G$.

Proof. Notice first that by Bazhenova's result 1 the set $\operatorname{Rat}(G)$ is a Boolean algebra. Obviously, the subgroup generated by $x_{1}$ and $x_{2}$ in $G$ is a free subgroup $F_{2}$ with basis $\left\{x_{1}, x_{2}\right\}$. If $w[G] \in \operatorname{Rat}(G)$ then $\bar{L}=w[G] \cap F_{2}$ is rational in $G$ by [1, hence by another Bazhenova's result [2] $\bar{L}$ is rational in $F_{2}$. By Corollary 4.9 the set $L=\bar{L} \cap X_{2}^{*}$ is rational in $X_{2}^{*}=\left\{x_{1}, x_{2}\right\}^{*}$ and so has a presentation of the form (11) in $X_{2}^{*}$. Since $w\left[F_{2}\right] \subseteq w[G]$ and the free decompositions of $F_{2}$ is induced from the free decomposition of $G$ one can complete the proof by an argument similar to the one from the proof of Theorem 5.6.

Corollary 5.8. Let $A$ and $B$ be groups containing elements of infinite order, and $G=A * B$. Let the abelianization $G_{a b}$ is infinite, and the rational sets $\operatorname{Rat}(A)$ and $\operatorname{Rat}(B)$ are Boolean algebras. Then for every proper word $w$ the set $w[G]$ is not rational in $G$.

Proof. Follows from Corollary 5.2 and Theorem 5.7

Corollary 5.9. Let $A$ and $B$ be infinite finitely generated abelian groups and $G=A * B$. Then for every proper word $w$ the set $w[G]$ is not rational in $G$.

Proof. Bazhenova showed in 22 that rational sets in finitely generated abelian groups form Boolean algebras. Now the result follows from 5.8

Theorem 5.7 and Corollaries 5.8 and 5.9 have far reaching generalizations. To explain we need the following simple but useful result.

Lemma 5.10. Suppose that a group $H$ admits a homomorphism onto a group $G$ in which every set $w[G]$ for a proper word $w$ is not rational. Then for every proper word $w$ the set $w[H]$ is not rational in $H$.

Proof. Suppose that $\varphi$ is a homomorphism of $H$ onto $G$. Since for every word $w$ we have $w[G]=\varphi(w[H])$, and a homomorphic image of any rational set is rational (see [8] $w[H] \in \operatorname{Rat}(H)$ implies that $w[G] \in \operatorname{Rat}(G)$ that contradicts our assumption. Hence, $w[H] \notin \operatorname{Rat}(H)$.

Corollary 5.11. Suppose that a group $H$ admits a homomorphism onto a free non-abelian group $F$. Then for every proper word $w$ the set $w[H]$ is not rational in $H$.

There are many classes of groups which have free non-abelian quotients. We list some of them below. 
Corollary 5.12. In the following groups $H$ for every proper word $w$ the set $w[H]$ is not rational:

1) Pure braid groups $P B_{n}$ for $n \geq 3$.

2) Non-abelian right angled Artin groups.

3) Finitely generated non-abelian residually free groups.

Proof. To prove 1) observe that a pure braid group $P B_{n}, n \geq 3$, has the group $P B_{3}$ as its epimorphic quotient (see [4, for example), and the group $P B_{3}$ is isomorphic to $F_{2} \times \mathbb{Z}$, so $P B_{n}, n \geq 3$, has the free group $F_{2}$ as its quotient.

To see 2) Let $G=G(\Gamma)$ be a non-abelian partially commutative group corresponding to a finite graph $\Gamma$. Then there are three vertices in $\Gamma$, say $v_{1}, v_{2}, v_{3}$ such that the complete subgraph $\Gamma_{0}$ of $\Gamma$ generated by these vertices is not a triangle. In particular, a partially commutative group $G_{0}=G\left(\Gamma_{0}\right)$ is either a free group $F_{3}$ (no edges in $\Gamma_{0}$ ), or $(\mathbb{Z} \times \mathbb{Z}) * \mathbb{Z}$ (only one edge in $\Gamma_{0}$ ), or $F_{2} \times \mathbb{Z}$ (precisely two edges in $\Gamma_{0}$ ). Notice that in all three cases the group $G\left(\Gamma_{0}\right)$ has $F_{2}$ as its epimorphic quotient. Now, it suffices to show that $G\left(\Gamma_{0}\right)$ is an epimorphic quotient of $G(\Gamma)$, which is obtained from $G(\Gamma)$ by adding to the standard presentation of $G(\Gamma)$ all the relations of the type $v=1$, where $v$ is a vertex of $\Gamma$ different from $v_{1}, v_{2}, v_{3}$. This shows that $F_{2}$ is a quotient of $G(\Gamma)$

By definition every non-abelian residually free group has a free non-abelian quotient, so 3) holds.

The following result shows that a wide class of free products of groups does not have rational proper verbal subsets.

Corollary 5.13. Suppose that $H=C * D$, where the factors $C, D$ are finitely generated groups with infinite abelianizations. Then for every proper word $w$ the set $w[H]$ is not rational in $H$.

\section{References}

[1] Bazhenova, G.A.: Closure of one class of groups under free products. Siberian Math. J., 41, 611-613 (2000).

[2] Bazhenova, G.A.: On rational sets in finitely generated nilpotent groups. Algebra and Log., 39, 215-223 (2000).

[3] Baumslag, B.: Intersections of finitely generated subgroups in free products. J. London Math. Soc., 41, 673-679 (1966).

[4] Birman, J.: Braids, Links and Mapping Class Groups. Princeton University Press, Annals of Math. Studies, Princeton (1974).

[5] Calegari, D.: SCL. Math. Soc. Japan Mem., vol. 20, Tokyo (2009).

[6] Calegari, D.: Quasimorphisms and laws. Algebr. Geom. Topol., 10, 215-217 (2010). 
[7] Gersten, S.: Cohomological lower bounds for isoperimetric functions on groups. Topology, 37, 1031-1072 (1998).

[8] Gilman, R.H.: Formal languages and infinite groups. In: Geometric and computational perspectives of infinite groups (Minneapolis, MN and New Brunswick, NJ, 1994), vol. 25 of DIMACS Ser. Discrete Math. Theoret. Comput. Sci., 27-51. Amer. Math. Soc., Providence, RI (1996).

[9] Gromov, M.: Volume and bounded cohomology. IHES Publ. Math., 56, 5-99 (1982).

[10] Gromov, M.: Asymptotic invariants of infinite groups. In: London Math. Soc. Lect. Notes Ser., vol. 182, Cambridge (1993).

[11] Larsen, M. and Shalev, A.: Word maps and Waring type problems. J. Amer. Math. Soc. 22, 437-466 (2009).

[12] Liebeck,M., O'Brian, E., Shalev A. and Tiep, P.: The Ore conjecture. J. European Math. Soc., 12, 939-1008 (2010).

[13] Magnus, W., Karrass, A. and Solitar, D.: Combinatorial Group Theory. Wiley Interscience, New York (1968).

[14] Makanin, G.S.: Equations in a free group (Russian), Izvestia Akademii Nauk SSSR, Ser. Matemat., 46, 1199-1273 (1982).

[15] Neumann, H.: Varieties of groups. Springer-Verlag, New York (1967).

[16] Ore, O.: Some remarks on commutators. Proc. Amer. Math. Soc. 2, 307-314 (1951).

[17] Razborov, A.: On the parametrization of solutions for equations in free groups. Int. J. Algebra and Comput., 3, 251-273 (1993).

[18] Rhemtulla, A.H.: A problem of bounded expressability in free products. Proc. Cambridge Philos. Soc., 64, 573-584 (1968).

[19] Rhemtulla, A.H.: Commutators of certain finitely generated soluble groups. Canad. J. Math., 21, 1160-1164 (1969).

[20] Roman'kov, V.A.: Width of verbal subgroups in solvable groups. Algebra and Log., 21, 41-49 (1982).

[21] Segal, D.: Words: notes on verbal width in groups, London Math. Soc. Lect. Notes Ser., vol. 361, Cambridge Univ. Press, Cambridge (2009).

[22] Shalev, A.: Word maps, conjugacy classes, and a noncommutative Waring-type theorem. Ann. of Math., 170, 1383-1416 (2009).

[23] Stroud, P.W.: Topics in the theory of verbal subgroups. PhD Thesis, Univ. of Cambridge, Cambridge (1966). 